\title{
GLOBAL BEHAVIOR OF A HIGHER-ORDER RATIONAL DIFFERENCE EQUATION
}

HONGJIAN XI AND TAIXIANG SUN

Received 17 January 2006; Revised 6 April 2006; Accepted 12 April 2006

We investigate in this paper the global behavior of the following difference equation: $x_{n+1}=\left(P_{k}\left(x_{n-i_{0}}, x_{n-i_{1}}, \ldots, x_{n-i_{2 k}}\right)+b\right) /\left(Q_{k}\left(x_{n-i_{0}}, x_{n-i_{1}}, \ldots, x_{n-i_{2 k}}\right)+b\right), n=0,1, \ldots$, under appropriate assumptions, where $b \in[0, \infty), k \geq 1, i_{0}, i_{1}, \ldots, i_{2 k} \in\{0,1, \ldots\}$ with $i_{0}<i_{1}<$ $\cdots<i_{2 k}$, the initial conditions $x_{i_{-2 k}}, x_{i_{-2 k}+1}, \ldots, x_{0} \in(0, \infty)$. We prove that unique equilib$\operatorname{rium} \bar{x}=1$ of that equation is globally asymptotically stable.

Copyright (c) $2006 \mathrm{H}$. Xi and T. Sun. This is an open access article distributed under the Creative Commons Attribution License, which permits unrestricted use, distribution, and reproduction in any medium, provided the original work is properly cited.

\section{Introduction}

For some difference equations, although their forms (or expressions) look very simple, it is extremely difficult to understand thoroughly the global behaviors of their solutions. Accordingly, one is often motivated to investigate the qualitative behaviors of difference equations (e.g., see $[2,3,6,9,10]$ ).

In [6], Ladas investigated the global asymptotic stability of the following rational difference equation:

(E1)

$$
x_{n+1}=\frac{x_{n}+x_{n-1} x_{n-2}}{x_{n} x_{n-1}+x_{n-2}}, \quad n=0,1, \ldots
$$

where the initial values $x_{-2}, x_{-1}, x_{0} \in \mathbb{R}_{+} \equiv(0,+\infty)$.

In [9], Nesemann utilized the strong negative feedback property of [1] to study the following difference equation:

(E2)

$$
x_{n+1}=\frac{x_{n-1}+x_{n} x_{n-2}}{x_{n} x_{n-1}+x_{n-2}}, \quad n=0,1, \ldots
$$

where the initial values $x_{-2}, x_{-1}, x_{0} \in \mathbb{R}_{+}$.

Hindawi Publishing Corporation

Advances in Difference Equations

Volume 2006, Article ID 27637, Pages 1-7

DOI 10.1155/ADE/2006/27637 
In [10], Papaschinopoulos and Schinas investigated the global asymptotic stability of the following nonlinear difference equation:

(E3)

$$
x_{n+1}=\frac{\sum_{i \in \mathbb{Z}_{k}-\{j-1, j\}} x_{n-i}+x_{n-j} x_{n-j+1}+1}{\sum_{i \in \mathbb{Z}_{k}} x_{n-i}}, \quad n=0,1, \ldots,
$$

where $k \in\{1,2,3, \ldots\},\{j, j-1\} \subset \mathbb{Z}_{k} \equiv\{0,1, \ldots, k\}$, and the initial values $x_{-k}, x_{-k+1}, \ldots$, $x_{0} \in \mathbb{R}_{+}$.

Recently, Li [7,8] studied the global asymptotic stability of the following two nonlinear difference equations:

(E4)

$$
x_{n+1}=\frac{x_{n-1} x_{n-2} x_{n-3}+x_{n-1}+x_{n-2}+x_{n-3}+a}{x_{n-1} x_{n-2}+x_{n-1} x_{n-3}+x_{n-2} x_{n-3}+1+a}, \quad n=0,1, \ldots
$$

(E5)

$$
x_{n+1}=\frac{x_{n} x_{n-1} x_{n-3}+x_{n}+x_{n-1}+x_{n-3}+a}{x_{n} x_{n-1}+x_{n} x_{n-3}+x_{n-1} x_{n-3}+1+a}, \quad n=0,1, \ldots
$$

where $a \in[0,+\infty)$ and the initial values $x_{-3}, x_{-2}, x_{-1}, x_{0} \in \mathbb{R}_{+}$.

Let $k \geq 1$ and $i_{0}, i_{1}, \ldots, i_{2 k} \in\{0,1, \ldots\}$ with $i_{0}<i_{1}<\cdots<i_{2 k}$. Let $P_{0}\left(x_{n-i_{0}}\right)=x_{n-i_{0}}$ and $Q_{0}\left(x_{n-i_{0}}\right)=1$, for any $1 \leq j \leq k$, let

$$
\begin{aligned}
P_{j}\left(x_{n-i_{0}}, \ldots, x_{n-i_{2 j}}\right)= & \left(x_{n-i_{2 j}} x_{n-i_{2 j-1}}+1\right) P_{j-1}\left(x_{n-i_{0}}, \ldots, x_{n-i_{2 j-2}}\right) \\
& +\left(x_{n-i_{2 j}}+x_{n-i_{2 j-1}}\right) Q_{j-1}\left(x_{n-i_{0}}, \ldots, x_{n-i_{2 j-2}}\right), \\
Q_{j}\left(x_{n-i_{0}}, \ldots, x_{n-i_{2 j}}\right)= & \left(x_{n-i_{2 j}} x_{n-i_{2 j-1}}+1\right) Q_{j-1}\left(x_{n-i_{0}}, \ldots, x_{n-i_{2 j-2}}\right) \\
& +\left(x_{n-i_{2 j}}+x_{n-i_{2 j-1}}\right) P_{j-1}\left(x_{n-i_{0}}, \ldots, x_{n-i_{2 j-2}}\right) .
\end{aligned}
$$

In this paper, we consider the following difference equation:

$$
x_{n+1}=\frac{P_{k}\left(x_{n-i_{0}}, x_{n-i_{1}}, \ldots, x_{n-i_{2 k}}\right)+b}{Q_{k}\left(x_{n-i_{0}}, x_{n-i_{1}}, \ldots, x_{n-i_{2 k}}\right)+b}, \quad n=0,1, \ldots,
$$

where $b \in[0, \infty)$ and the initial conditions $x_{-i_{2 k}}, x_{-i_{2 k}+1}, \ldots, x_{0} \in(0, \infty)$.

It is easy to see that the positive equilibrium $\bar{x}$ of (1.7) satisfies

$$
\begin{aligned}
\bar{x} & =\frac{P_{k}(\bar{x}, \bar{x}, \ldots, \bar{x})+b}{Q_{k}(\bar{x}, \bar{x}, \ldots, \bar{x})+b} \\
& =\frac{\left(\bar{x}^{2}+1\right) P_{k-1}(\bar{x}, \bar{x}, \ldots, \bar{x})+2 \bar{x} Q_{k-1}(\bar{x}, \bar{x}, \ldots, \bar{x})+b}{\left(\bar{x}^{2}+1\right) Q_{k-1}(\bar{x}, \bar{x}, \ldots, \bar{x})+2 \bar{x} P_{k-1}(\bar{x}, \bar{x}, \ldots, \bar{x})+b} .
\end{aligned}
$$


Thus, we have

$$
(\bar{x}-1)\left[\left(\bar{x}^{2}+\bar{x}\right) Q_{k-1}(\bar{x}, \bar{x}, \ldots, \bar{x})+(\bar{x}+1) P_{k-1}(\bar{x}, \bar{x}, \ldots, \bar{x})+b\right]=0,
$$

from which one can see that (1.7) has the unique positive equilibrium $\bar{x}=1$.

Remark 1.1. Let $k=1$, then (1.7) is (1.4) when $\left(i_{0}, i_{1}, i_{2}\right)=(1,2,3)$ and is (1.5) when $\left(i_{0}, i_{1}, i_{2}\right)=(0,1,3)$.

\section{Properties of positive solutions of (1.7)}

In this section, we will study properties of positive solutions of (1.7). Since

$$
\begin{aligned}
P_{k}\left(x_{n-i_{0}}, x_{n-i_{1}}, \ldots, x_{n-i_{2 k}}\right)-Q_{k}\left(x_{n-i_{0}}, x_{n-i_{1}}, \ldots, x_{n-i_{2 k}}\right) \\
\quad=\left(x_{n-i_{2 k}}-1\right)\left(x_{n-i_{2 k-1}}-1\right)\left[P_{k-1}\left(x_{n-i_{0}}, \ldots, x_{n-i_{2 k-2}}\right)-Q_{k-1}\left(x_{n-i_{0}}, \ldots, x_{n-i_{2 k-2}}\right)\right] \\
\quad=\cdots \\
\quad=\left(x_{n-i_{2 k}}-1\right)\left(x_{n-i_{2 k-1}}-1\right) \cdots\left(x_{n-i_{2}}-1\right)\left(x_{n-i_{1}}-1\right)\left[P_{0}\left(x_{n-i_{0}}\right)-Q_{0}\left(x_{n-i_{0}}\right)\right] \\
\quad=\left(x_{n-i_{0}}-1\right)\left(x_{n-i_{1}}-1\right) \cdots\left(x_{n-i_{2 k}}-1\right),
\end{aligned}
$$

it follows from (1.7) that for any $n \geq 0$,

$$
x_{n+1}-1=\frac{\left(x_{n-i_{0}}-1\right)\left(x_{n-i_{1}}-1\right) \cdots\left(x_{n-i_{2 k}}-1\right)}{Q_{k}\left(x_{n-i_{0}}, x_{n-i_{1}}, \ldots, x_{n-i_{2 k}}\right)+b} .
$$

Definition 2.1. Let $\left\{x_{n}\right\}_{n=-i_{2 k}}^{\infty}$ be a solution of (1.7) and $\left\{a_{n}\right\}_{n=-i_{2 k}}^{\infty}$ a sequence with $a_{n} \in$ $\{-1,0,1\}$ for every $n \geq-i_{2 k} .\left\{a_{n}\right\}_{n=-i_{2 k}}^{\infty}$ is called itinerary of $\left\{x_{n}\right\}_{n=-i_{2 k}}^{\infty}$ if $a_{n}=-1$ when $x_{n}<1, a_{n}=0$ when $x_{n}=1$, and $a_{n}=1$ when $x_{n}>1$.

From (2.2), we get the following.

Proposition 2.2. Let $\left\{x_{n}\right\}_{n=-i_{2 k}}^{\infty}$ be a solution of (1.7) whose itinerary is $\left\{a_{n}\right\}_{n=-i_{2 k}}^{\infty}$, then $a_{n+1}=a_{n-i_{0}} a_{n-i_{1}} \cdots a_{n-i_{2 k}}$ for any $n \geq 0$.

Proposition 2.3. Let $\left\{x_{n}\right\}_{n=-i_{2 k}}^{\infty}$ be a solution of (1.7), then it follows that $x_{n} \neq 1$ for any $n \geq 1 \Leftrightarrow \prod_{j=0}^{i_{2 k}}\left(x_{-j}-1\right) \neq 0$.

Proof. Let itinerary of $\left\{x_{n}\right\}_{n=-i_{2 k}}^{\infty}$ be $\left\{a_{n}\right\}_{n=-i_{2 k}}^{\infty}$, then it follows from Proposition 2.2 that $x_{n} \neq 1$ for any $n \geq 1 \Leftrightarrow a_{n} \neq 0$ for any $n \geq 1 \Leftrightarrow \prod_{j=0}^{i_{2 k}} a_{-j} \neq 0 \Leftrightarrow \prod_{j=0}^{i_{2 k}}\left(x_{-j}-1\right) \neq 0$.

Proposition 2.4. If $\operatorname{gcd}\left(i_{s}+1, i_{2 k}+1\right)=1$ for some $s \in\{0,1, \ldots, 2 k-1\}$, then a positive solution $\left\{x_{n}\right\}_{n=-i_{2 k}}^{\infty}$ of (1.7) is eventually equal to $1 \Leftrightarrow x_{p}=1$ for some $p \geq-i_{2 k}$.

Proof. "If" part is obvious.

"Only if" part. If $x_{p}=1$ for some $p \geq-i_{2 k}$, then $a_{p}=0$, where $\left\{a_{n}\right\}_{n=-i_{2 k}}^{\infty}$ is itinerary of $\left\{x_{n}\right\}_{n=-i_{2 k}}^{\infty}$. By Proposition 2.2, we have $a_{j\left(i_{2 k}+1\right)+p}=a_{j\left(i_{s}+1\right)+p}=0$ for any $j \geq 0$. Since $\operatorname{gcd}\left(i_{s}+1, i_{2 k}+1\right)=1$, we see that for any $t \in\left\{0,1, \ldots, i_{2 k}\right\}$, there exist $j_{t} \in\left\{1,2, \ldots, i_{2 k}+1\right\}$ 
4 Global behavior of a difference equation

and $m_{t} \in\left\{0,1, \ldots, i_{s}+1\right\}$ such that

$$
j_{t}\left(i_{s}+1\right)=m_{t}\left(i_{2 k}+1\right)+t
$$

Together with Proposition 2.2, it follows that

$$
a_{\left(i_{s}+1\right)\left(i_{2 k}+1\right)+t+p}=0 .
$$

Again by Proposition 2.2, we have $a_{n}=0$ for any $n \geq\left(i_{s}+1\right)\left(i_{2 k}+1\right)+p$, which implies $x_{n}=1$ for any $n \geq\left(i_{s}+1\right)\left(i_{2 k}+1\right)+p$.

Example 2.5. Consider the equation

$$
x_{n+1}=\frac{x_{n-i_{0}} x_{n-i_{1}} x_{n-3}+x_{n-i_{0}}+x_{n-i_{1}}+x_{n-3}+b}{x_{n-i_{0}} x_{n-i_{1}}+x_{n-i_{0}} x_{n-3}+x_{n-i_{1}} x_{n-3}+1+b}, \quad n=0,1, \ldots,
$$

where $b \in[0,+\infty), 0 \leq i_{0}<i_{1}<3$, and the initial values $x_{-3}, x_{-2}, x_{-1}, x_{0} \in \mathbb{R}_{+}$. Let $\left\{x_{n}\right\}_{n=-3}^{\infty}$ be a solution of (2.5) whose itinerary is $\left\{a_{n}\right\}_{n=-3}^{\infty}$, then the following hold.

(1) If $\left(i_{0}, i_{1}\right) \in\{(0,1),(1,2)\}$ and $\left\{x_{n}\right\}_{n=-3}^{\infty}$ is not eventually equal to 1 , then $\left\{a_{n}\right\}_{n=-3}^{\infty}$ is a periodic sequence of period 7 .

(2) If $\left(i_{0}, i_{1}\right)=(0,2)$ and $\left\{x_{n}\right\}_{n=-3}^{\infty}$ is not eventually equal to 1 , then $\left\{a_{n}\right\}_{n=-3}^{\infty}$ is a periodic sequence of period 6 .

(3) $x_{n} \neq 1$ for any $n \geq 1 \Leftrightarrow \prod_{j=-3}^{0}\left(x_{j}-1\right) \neq 0$.

(4) $\left\{x_{n}\right\}_{n=-3}^{\infty}$ is eventually equal to $1 \Leftrightarrow x_{p}=1$ for some $p \geq-3$.

Proof. (1) If $\left(i_{0}, i_{1}\right)=(0,1)$, then from Proposition 2.2, it follows that for any $n \geq 0$,

$$
\begin{aligned}
a_{n+4} & =a_{n+3} a_{n+2} a_{n}=a_{n+2} a_{n+1} a_{n-1} a_{n+2} a_{n} \\
& =a_{n+1} a_{n} a_{n-1}=a_{n} a_{n-1} a_{n-3} a_{n} a_{n-1} \\
& =a_{n-3} .
\end{aligned}
$$

If $\left(i_{0}, i_{1}\right)=(1,2)$, then in a similar fashion, it is true that $a_{n+4}=a_{n-3}$ for any $n \geq 0$.

(2) If $\left(i_{0}, i_{1}\right)=(0,2)$, then from Proposition 2.2, it follows that for any $n \geq 0$,

$$
\begin{aligned}
a_{n+3} & =a_{n+2} a_{n} a_{n-1}=a_{n+1} a_{n-1} a_{n-2} a_{n} a_{n-1} \\
& =a_{n+1} a_{n} a_{n-2}=a_{n} a_{n-2} a_{n-3} a_{n} a_{n-2} \\
& =a_{n-3} .
\end{aligned}
$$

(3) It follows from Proposition 2.3.

(4) It follows from Proposition 2.4 since either $\operatorname{gcd}\left(i_{0}+1,4\right)=1$ or $\operatorname{gcd}\left(i_{1}+1,4\right)=1$.

\section{Global asymptotic stability of (1.7)}

In this section, we will study global asymptotic stability of (1.7). To do this, we need the following lemmas. 
LemMA 3.1. Let $\left(y_{0}, y_{1}, \ldots, y_{i_{2 k}}\right) \in \mathbb{R}_{+}^{i_{2 k}+1}-\{(1,1, \ldots, 1)\}$ and $M=\max \left\{y_{j}, 1 / y_{j} \mid 0 \leq j \leq\right.$ $\left.i_{2 k}\right\}$, then

$$
\frac{1}{M}<\frac{P_{k}\left(y_{i_{0}}, y_{i_{1}}, \ldots, y_{i_{2 k}}\right)}{Q_{k}\left(y_{i_{0}}, y_{i_{1}}, \ldots, y_{i_{2 k}}\right)}<M
$$

Proof. Since $\left(y_{0}, y_{1}, \ldots, y_{i_{2 k}}\right) \in \mathbb{R}_{+}^{i_{2 k}+1}-\{(1,1, \ldots, 1)\}$ and $M=\max \left\{y_{j}, 1 / y_{j} \mid 0 \leq j \leq i_{2 k}\right\}$, we have $M>1$ and either $M \geq a>1 / M$ or $M>a \geq 1 / M$ for any $a \in\left\{y_{j}, 1 / y_{j} \mid 0 \leq j \leq\right.$ $\left.i_{2 k}\right\}$.

It is easy to verify that

$$
\begin{aligned}
P_{1}\left(y_{i_{0}}, y_{i_{1}}, y_{i_{2}}\right) & =\left(y_{i_{1}} y_{i_{2}}+1\right) y_{i_{0}}+\left(y_{i_{1}}+y_{i_{2}}\right) \\
& <\left(y_{i_{1}} y_{i_{2}}+1\right) M+\left(y_{i_{1}}+y_{i_{2}}\right) y_{i_{0}} M \\
& =Q_{1}\left(y_{i_{0}}, y_{i_{1}}, y_{i_{2}}\right) M, \\
P_{1}\left(y_{i_{0}}, y_{i_{1}}, y_{i_{2}}\right) M & =\left[\left(y_{i_{1}} y_{i_{2}}+1\right) y_{i_{0}}+\left(y_{i_{1}}+y_{i_{2}}\right)\right] M \\
& >\left(y_{i_{1}} y_{i_{2}}+1\right)+\left(y_{i_{1}}+y_{i_{2}}\right) y_{i_{0}} \\
& =Q_{1}\left(y_{i_{0}}, y_{i_{1}}, y_{i_{2}}\right) .
\end{aligned}
$$

From that we have

$$
\begin{aligned}
P_{2}\left(y_{i_{0}}, y_{i_{1}}, y_{i_{2}}, y_{i_{3}}, y_{i_{4}}\right) & =\left(y_{i_{3}} y_{i_{4}}+1\right) P_{1}\left(y_{i_{0}}, y_{i_{1}}, y_{i_{2}}\right)+\left(y_{i_{3}}+y_{i_{4}}\right) Q_{1}\left(y_{i_{0}}, y_{i_{1}}, y_{i_{2}}\right) \\
& <\left(y_{i_{3}} y_{i_{4}}+1\right) Q_{1}\left(y_{i_{0}}, y_{i_{1}}, y_{i_{2}}\right) M+\left(y_{i_{3}}+y_{i_{4}}\right) P_{1}\left(y_{i_{0}}, y_{i_{1}}, y_{i_{2}}\right) M \\
& =Q_{2}\left(y_{i_{0}}, y_{i_{1}}, y_{i_{2}}, y_{i_{3}}, y_{i_{4}}\right) M, \\
P_{2}\left(y_{i_{0}}, y_{i_{1}}, y_{i_{2}}, y_{i_{3}}, y_{i_{4}}\right) M & =\left[\left(y_{i_{3}} y_{i_{4}}+1\right) P_{1}\left(y_{i_{0}}, y_{i_{1}}, y_{i_{2}}\right)+\left(y_{i_{3}}+y_{i_{4}}\right) Q_{1}\left(y_{i_{0}}, y_{i_{1}}, y_{i_{2}}\right)\right] M \\
& >\left(y_{i_{3}} y_{i_{4}}+1\right) Q_{1}\left(y_{i_{0}}, y_{i_{1}}, y_{i_{2}}\right)+\left(y_{i_{3}}+y_{i_{4}}\right) P_{1}\left(y_{i_{0}}, y_{i_{1}}, y_{i_{2}}\right) \\
& =Q_{2}\left(y_{i_{0}}, y_{i_{1}}, y_{i_{2}}, y_{i_{3}}, y_{i_{4}}\right) .
\end{aligned}
$$

By induction, we have that for any $1 \leq j \leq k$,

$$
\begin{gathered}
P_{j}\left(y_{i_{0}}, y_{i_{1}}, \ldots, y_{i_{2 j}}\right)<Q_{j}\left(y_{i_{0}}, y_{i_{1}}, \ldots, y_{i_{2 j}}\right) M, \\
P_{j}\left(y_{i_{0}}, y_{i_{1}}, \ldots, y_{i_{2 j}}\right) M>Q_{j}\left(y_{i_{0}}, y_{i_{1}}, \ldots, y_{i_{2 j}}\right) .
\end{gathered}
$$

Thus

$$
\frac{1}{M}<\frac{P_{k}\left(y_{i_{0}}, y_{i_{1}}, \ldots, y_{i_{2 k}}\right)}{Q_{k}\left(y_{i_{0}}, y_{i_{1}}, \ldots, y_{i_{2 k}}\right)}<M
$$


Let $n$ be a positive integer and let $\rho$ denote the part-metric on $\mathbb{R}_{+}^{n}$ (see [11]) which is defined by

$$
\rho(x, y)=-\log \min \left\{\frac{x_{i}}{y_{i}}, \frac{y_{i}}{x_{i}} \mid 1 \leq i \leq n\right\} \quad \text { for } x=\left(x_{1}, \ldots, x_{n}\right), y=\left(y_{1}, \ldots, y_{n}\right) \in \mathbb{R}_{+}^{n} .
$$

It was shown by Thompson [11] that $\left(\mathbb{R}_{+}^{n}, \rho\right)$ is a complete metric space. In [4], Krause and Nussbaum proved that the distances indicated by the part-metric and by the Euclidean norm are equivalent on $\mathbb{R}_{+}^{n}$.

Lemma 3.2 [5]. Let $T: \mathbb{R}_{+}^{n} \rightarrow \mathbb{R}_{+}^{n}$ be a continuous mapping with unique fixed point $x^{*} \in \mathbb{R}_{+}^{n}$. Suppose that there exists some $l \geq 1$ such that for the part-metric $\rho$,

$$
\rho\left(T^{l} x, x^{*}\right)<\rho\left(x, x^{*}\right) \quad \forall x \neq x^{*} .
$$

Then $x^{*}$ is globally asymptotically stable.

Theorem 3.3. The unique equilibrium $\bar{x}=1$ of (1.7) is globally asymptotically stable.

Proof. Let $\left\{x_{n}\right\}_{n=-i_{2 k}}^{\infty}$ be a solution of (1.7) with initial conditions $x_{-i_{2 k}}, x_{-i_{2 k}+1}, \ldots, x_{0} \in$ $\mathbb{R}_{+}^{i_{2 k}+1}$ such that $\left\{x_{n}\right\}_{n=-i_{2 k}}^{\infty}$ is not eventually equal to 1 since otherwise there is nothing to show. Denoted by $T: \mathbb{R}_{+}^{i_{2 k}+1} \rightarrow \mathbb{R}_{+}^{i_{2 k}+1}$ the mapping

$$
T\left(x_{n-i_{2 k}}, x_{n-i_{2 k}+1}, \ldots, x_{n}\right)=\left(x_{n-i_{2 k}+1}, x_{n-i_{2 k}+2}, \ldots, x_{n}, \frac{P_{k}\left(x_{n-i_{0}}, x_{n-i_{1}}, \ldots, x_{n-i_{2 k}}\right)+b}{Q_{k}\left(x_{n-i_{0}}, x_{n-i_{1}}, \ldots, x_{n-i_{2 k}}\right)+b}\right) .
$$

Then solution $\left\{x_{n}\right\}_{n=-i_{2 k}}^{\infty}$ of (1.7) is represented by the first component of the solution $\left\{y_{n}\right\}_{n=0}^{\infty}$ of the system $y_{n+1}=T y_{n}$ with initial condition $y_{0}=\left(x_{-i_{2 k}}, x_{-i_{2 k}+1}, \ldots, x_{0}\right)$. It follows from Lemma 3.1 that for all $n \geq 0$, the following inequalities hold:

$$
\min \left\{x_{n-i}, \frac{1}{x_{n-i}} \mid 0 \leq i \leq i_{2 k}\right\}<x_{n+1}<\max \left\{x_{n-i}, \frac{1}{x_{n-i}} \mid 0 \leq i \leq i_{2 k}\right\}
$$

Inductively, we obtain that for all $n \geq 0$ and all $1 \leq j \leq i_{2 k}+1$,

$$
\min \left\{x_{n-i}, \frac{1}{x_{n-i}} \mid 0 \leq i \leq i_{2 k}\right\}<x_{n+j}<\max \left\{x_{n-i}, \frac{1}{x_{n-i}} \mid 0 \leq i \leq i_{2 k}\right\}
$$

from which it follows that

$$
\min \left\{x_{n-i}, \frac{1}{x_{n-i}} \mid 0 \leq i \leq i_{2 k}\right\}<\min \left\{x_{n+i}, \frac{1}{x_{n+i}} \mid 1 \leq i \leq i_{2 k}+1\right\}
$$


Thus, for $x^{*}=(1,1, \ldots, 1)$ and the part-metric $\rho$ of $\mathbb{R}_{+}^{i_{2 k}+1}$, we have

$$
\begin{aligned}
\rho\left(T^{i_{2 k}+1}\left(y_{n}\right), x^{*}\right) & =-\log \min \left\{x_{n+i}, \frac{1}{x_{n+i}} \| 1 \leq i \leq i_{2 k}+1\right\} \\
& <-\log \min \left\{x_{n-i}, \frac{1}{x_{n-i}} \mid 0 \leq i \leq i_{2 k}\right\} \\
& =\rho\left(y_{n}, x^{*}\right)
\end{aligned}
$$

for all $n \geq 0$. It follows from Lemma 3.2 that the positive equilibrium $\bar{x}=1$ of (1.7) is globally asymptotically stable.

\section{Acknowledgments}

Project supported by NNSF of China $(10461001,10361001)$ and NSF of Guangxi (0640205).

\section{References}

[1] A. M. Amleh, N. Kruse, and G. Ladas, On a class of difference equations with strong negative feedback, Journal of Difference Equations and Applications 5 (1999), no. 6, 497-515.

[2] C. Çinar, On the positive solutions of the difference equation $x_{n+1}=\alpha x_{n-1} /\left(1+b x_{n} x_{n-1}\right)$, Applied Mathematics and Computation 156 (2004), no. 2, 587-590.

[3] H. M. El-Owaidy, A. M. Ahmed, and M. S. Mousa, On the recursive sequences $x_{n+1}=$ $-\alpha x_{n-1} /\left(\beta \pm x_{n}\right)$, Applied Mathematics and Computation 145 (2003), no. 2-3, 747-753.

[4] U. Krause and R. D. Nussbaum, A limit set trichotomy for self-mappings of normal cones in Banach spaces, Nonlinear Analysis 20 (1993), no. 7, 855-870.

[5] N. Kruse and T. Nesemann, Global asymptotic stability in some discrete dynamical systems, Journal of Mathematical Analysis and Applications 235 (1999), no. 1, 151-158.

[6] G. Ladas, Open problems and conjectures, Journal of Difference Equations and Applications 4 (1998), no. 1, 497-499.

[7] X. Li, Global behavior for a fourth-order rational difference equation, Journal of Mathematical Analysis and Applications 312 (2005), no. 2, 555-563.

[8] __ Qualitative properties for a fourth-order rational difference equation, Journal of Mathematical Analysis and Applications 311 (2005), no. 1, 103-111.

[9] T. Nesemann, Positive nonlinear difference equations: some results and applications, Nonlinear Analysis 47 (2001), no. 7, 4707-4717.

[10] G. Papaschinopoulos and C. J. Schinas, Global asymptotic stability and oscillation of a family of difference equations, Journal of Mathematical Analysis and Applications 294 (2004), no. 2, 614620.

[11] A. C. Thompson, On certain contraction mappings in a partially ordered vector space, Proceedings of the American Mathematical Society 14 (1963), no. 3, 438-443.

Hongjian Xi: Department of Mathematics, Guangxi College of Finance and Economics, Nanning, Guangxi 530004, China

E-mail address: xhongjian@263.com

Taixiang Sun: Department of Mathematics, College of Mathematics and Information Science, Guangxi University, Nanning, Guangxi 530004, China

E-mail address: stx1963@163.com 